\title{
Basilar-type migraine patients responsive to lamotrigine: a 5-year follow-up
}

\author{
D. Cologno $\cdot$ F. d'Onofrio $\cdot$ O. Castriota \\ V. Petretta $\cdot$ G. Casucci $\cdot$ A. Russo $\cdot$ \\ G. Bussone
}

(C) Springer-Verlag Italia 2013

\begin{abstract}
Five years ago we reported the case of three patients affected by basilar-type migraine (BM) responsive to lamotrigine. At that time, proven treatment options for $\mathrm{BM}$ are rather limited and lamotrigine has been tested in BM patients because it was a widely tested treatment for migraine with aura. That positive 1-year experience leaded us to suggest that lamotrigine could be a preventive therapeutic option for BM patients, with and without menstruation association. We now report the five-year follow-up of the same patients to confirm and underlie the possible role of lamotrigine to induce BM attacks remission.
\end{abstract}

Keywords Basilar-type migraine - Lamotrigine ·

Follow-up

D. Cologno $(\varangle) \cdot$ O. Castriota

Department of Neuroscience, Institute of Clinical

Neurophysiology, Azienda Ospedaliero-Universitaria,

Ospedali Riuniti, Foggia, Italy

e-mail: danielacologno@virgilio.it

F. d'Onofrio · V. Petretta

Institute of Neurology San G. Moscati Hospital, Avellino, Italy

G. Casucci

S. Francesco Nursing Home, Telese Terme, BN, Italy

A. Russo

Department of Neurology, Headache Center, Second University of Naples, Naples, Italy

\footnotetext{
A. Russo

Institute for Diagnosis and Care 'Hermitage Capodimonte',

Naples, Italy

G. Bussone

Clinical Neuroscience and Headache Unit, C. Besta Neurological Institute and Foundation, Milan, Italy
}

\section{Case reports}

Three women, patient 1 (age 37), patient 2 (age 43) and patient 3 (age 43), have been followed in the last 5 years at the Headache Centre of Avellino. Migraine diagnosis has been confirmed: all three had headaches with aura fulfilling the ICHD-II criteria [1] for BM. Patients 1 and 2 are sisters. As in the previous report [2], no attacks of typical migraine (with or without aura) occurred in the sisters in the last 5 years, while patient 3 continued to have attacks fulfilling ICHD-II criteria [1] for migraine without aura. None of the women reported other medical conditions.

The aura and pain had same features of the past. All women described a typical aura as characterized by slowly worsening bilateral blindness and bilateral hand paraesthesia spreading to the mouth and tongue (tongue in patient 3). Patient 2 also reported speech disturbances. In all cases, these symptoms progressed over about $10 \mathrm{~min}$ and, 10-20 min after onset, severe headache developed, which was bilateral in patient 1 and unilateral in patients 2 and 3. The pain lasted 5-24 h and was accompanied by nausea, photophobia and phonophobia, in two patients by vomiting. At the time of first observation, BM frequency in the two sisters (patient 1 and 2) had been 1 per month with attacks always occurred in association with menstruation (often on the day preceding bleeding onset) and it was very low (1 per 4-5 months) in patient 3 without association with menstruation. In this patient, discontinuation of oral contraceptives used over the previous 3 years had no effect on the BM attack frequency. None of the women were taking preventive medication for their headache when they came to us for the first time.

\section{Follow-up period}

In this follow-up period (2007-2012), all three patients had no other pregnancies and have not been taken oral 
contraceptives. Patient 1 continued to take lamotrigine at the same dosage $(100 \mathrm{mg} /$ day $)$ from the last observation (2007) until 2009 with a complete remission of BM attacks. She decided by self to discontinue the drug and for 3 years, up to May 2012, she was pain-free. BM attacks reappeared 6 months ago always related to menstruation. No significant medical events have been related to complete remission before and after reappearance of BM attacks. She referred again to headache centre and she started to take magnesium ( $4.5 \mathrm{~g} /$ day) 3 days before menstruation until they stopped. At the last observation (five menstruations), she did not refer anymore BM attacks. As patient 1, her sister (patient 2) took lamotrigine for all the period since her first observation. She was in menopausal period from August 2012 and on the next month she decided spontaneously to discontinue lamotrigine. BM attacks reappeared immediately with a higher monthly frequency than the past (2-3 attacks per month). We recommended to take again lamotrigine at the same dosage $(100 \mathrm{mg} /$ day $)$ with a fast BM attacks remission. About patient 3, she has been recalled by phone 3 months ago and invited to come to our headache centre. She reported to discontinue lamotrigine 1 year before with no reappearance of BM attacks.

\section{Investigations}

Neurological examination was normal in all three patients at follow-up ambulatory visit. The same investigations of the first observation have been performed at follow-up (routine blood and urine tests, ophthalmological and cardiological evaluations, colour Doppler ultrasonography of the supra-aortic vessels, standard EEG, gadoliniumenhanced cerebral MRI and angio-MRI) and they were all normal. Only brain SPECT has not been performed again.

\section{Discussion}

In the last 5 years, reports and clinical or therapeutic data about $\mathrm{BM}$ have been rare. It remains uncertain whether BM is a subtype of migraine with aura (MA) or a distinct phenotype or genotype. Olesen et al. [3] referred that BM occurred in $10 \%$ of MA patients with no firm clinical, epidemiological or genetic evidence that BM is an independent disease entity, different from MA. BM and MA show many similarities also from a therapeutical approach. This consideration addressed us in the past to use in these three of our BM patients lamotrigine, effective drug in preventing migraine aura and cortical spreading depression and in reducing migraine headache frequency [4]. After open study by Pascual et al. [5] on lamotrigine use for the preventive treatment of selected patients with disturbing migraine auras, including those with $\mathrm{BM}$ and hemiplegic migraine, no other clinical trials have been reported in the literature. Other anticonvulsant drugs have been tested in BM such as sodium valproate [6] and topiramate in a child population [7] with a good response at both 25 and $100 \mathrm{mg}$ doses. More recently, acetazolamide [8] relieved BM attacks in a reported patient with both BM and episodic ataxia type 2 and a novel nonsense mutation in CACNA1A gene at codon position 583 .

As during first observation, also in the follow-up period our report provided evidence on usefulness of lamotrigine in BM. In our three patients, lamotrigine resulted in complete remission of BM and was well tolerated and without adverse events over the whole follow-up period of treatment, confirming that the drug could be tried as a prophylactic in patients with $\mathrm{BM}$ who suffer a severe or distressing aura. Over the time, the BM attacks in two of our patients have been always associated with menstruation, suggesting the possibility that BM may exist also as a menstrual subtype. Nowadays, no other reports have been published on the use of lamotrigine in menstrual migraine. The rarity of scientific reports on BM about its clinical features and therapeutic approaches derived from its likely very low population prevalence. This could be a hallmark to reconsider BM as a clinical entity or as a subtype of MA associated or not to menstruation.

Conflict of interest The author certifies that there is no actual or potential conflict of interest in relation to this article.

\section{References}

1. Headache Classification Subcommittee of the International Headache Society (2004) The International Classification of Headache Disorders, 2nd edn. Cephalalgia 24(Suppl 1):1-160

2. d'Onofrio F, Cologno D, Petretta V, Casucci G, Bussone G (2007) Basilar-type migraine responsive to lamotrigine: three case report. Neurol Sci 28:S239-S241

3. Kirchmann M, Thomsen LL, Olesen J (2006) Basilar-type migraine: clinical, epidemiologic, and genetic features. Neurology 66(6):880-886

4. Lampl C, Katsarava Z, Diener HC, Limmroth V (2005) Lamotrigine reduces migraine aura and migraine attacks in patients with migraine with aura. J Neurosurg Psychiatry 76:1730-1732

5. Pascual J, Caminero AB, Mateos V et al (2004) Preventing disturbing migraine aura with lamotrigine: an open study. Headache 44:1024-1028

6. Kozubski W (2005) Basilar-type migraine: pathophysiology, symptoms and signs, and treatment. Neurol Neurochir Pol 39(4 Suppl 1):S65-S67

7. Lewis D, Paradiso E (2007) A double-blind, dose comparison study of topiramate for prophylaxis of basilar-type migraine in children: a pilot study. Headache 47(10):1409-1417

8. Robbins MS, Lipton RB, Laureta EC, Grosberg BM (2009) CACNA1A nonsense mutation is associated with basilar-type migraine and episodic ataxia type 2. Headache 49(7):1042-1046 